\title{
Idiopathic hypercalciuria in children with urinary tract infection
}

The presence of hypercalciuria in children with urinary tract infection in our population is the focus of great interest. When the amount of calcium excreted into the urine is larger than physiological values, it should be considered an important risk factor since this condition may predispose to concurrent medical events, such as hematuria, kidney stones and bone mass reduction. It is therefore necessary to address this subject in depth and take it into account when managing patients with recurrent urinary tract infection. Patients with high urinary calcium values (above physiological values) require to follow a normal protein, low sodium diet and have adequate regular controls. Sometimes these measures are not enough to achieve normal urinary calcium values and a more specific treatment should be added, such as sodium citrate and hydrochlorothiazide + amiloride.

In this issue of Archivos Argentinos de Pediatría (see page 428), Balestracci, et al. reviewed the rate of children with urinary tract infection and idiopathic hypercalciuria. The authors also looked into whether the presence of vesicoureteral reflux was related to such association. Their study was conducted on 136 patients seen at the Department of Nephrology of Hospital Pedro de Elizalde, in the city of Buenos Aires. The authors found a $19.8 \%$ prevalence of urinary tract infections, which is higher than that published by other authors from different countries who conducted studies on asymptomatic children with a history of urinary tract infection (range: 5\%-10\%), but is relatively low in relation to children with symptomatic urinary tract infection.

Since the prevalence of idiopathic hypercalciuria is higher when it is accompanied by urinary tract infection, it is important to find out whether patients had signs and symptoms of urinary tract infection at the time of diagnosis. Although this information is not clearly stated in the article, it is assumed that included patients did not have urinary tract infection at the time of the study. If this were the case, I would like to emphasize that it is necessary to consider that the prevalence of urinary tract infection found by the authors is high when compared to the values published by other studies.

The authors did not find a significant difference in the prevalence of idiopathic hypercalciuria in children with and without vesicoureteral reflux. However, when the prevalence was analyzed in relation to firsttime or recurrent urinary tract infection, a significant difference was observed, with a higher prevalence of idiopathic hypercalciuria in children with recurrent urinary tract infections, although they did not have vesicoureteral reflux. They also found a statistically significant relationship between this group of patients and a high sodium intake.

This article provides interesting data that emphasize the importance of determining urinary calcium levels in patients with recurrent urinary tract infection, with or without confirmed vesicoureteral reflux.

María Gracia Caletti, M.D. Consultant, Department of Nephrology, Hospital de Pediatría "Prof. Dr. Juan P. Garrahan" Buenos Aires http:/ /dx.doi.org/10.5546/aap.2014.eng.396 